\title{
Estado Nutricional y Conductas Asociadas a la Nutrición en Escolares
}

\author{
SONIA JAIMOVICH P. ${ }^{1}$, CECILIA CAMPOS S. ${ }^{1}$, MARÍA SYLVIA CAMPOS S. ${ }^{2}$, \\ JEAN MOORE B. ${ }^{3}$, LISA PAWLOSKI R. ${ }^{4}$, KATHLEEN GAFFNEY F. ${ }^{5}$ \\ 1. Enfermera, Magister en Salud Pública, Profesor Titular Escuela de Enfermería Pontificia Universidad Católica de Chile. \\ 2. Enfermera Matrona, Magister en Nutrición, Profesor Asociada, Escuela de Enfermería Pontificia Universidad Católica \\ de Chile. \\ 3. PhD, RN, School of Nursing, George Mason University, Fairfax, VA USA. \\ 4. PhD, Professor and Chair Dept. of Global and Community Health, George Mason University, Fairfax, VA USA. \\ 5. PhD, RN, Professor School of Nursing, George Mason University, Fairfax, VA USA.
}

\begin{abstract}
\section{Nutritional Status and Nutrition-Related Behavior in School-Agers}

Objectives: To analyze nutritional self-care behavior and nutritional status among children in two urban-zone schools in Santiago, Chile. Population and Methods: Analysis of data obtained in a transversal descriptive study of 207 volunteer children aged 9-15 y.o. in 2008, used to validate the Moore Index NutritionChild (MINC)(C. Results: For the behaviors under review, average test score among children was only $63 \%$ of expected $(210 \mathrm{pts})$. Least favorable responses relate to obtaining healthy eating information through various media. Most favorable relate to food hygiene. Behavioral comparison according to nutritional status shows no correlation; average score for the full scale, for all children, was 132,2 $\mathrm{SD} 22.1$ (One-way Anova). Overweight and obese children showed no significant differences in scores. Discussion and conclusion: In order to decrease the prevalence of obesity, results suggest intervention focused on acquisition of knowledge and searching of information.
\end{abstract}

(Key words: Nutrition-Related Behavior, Nutritional Status, schoolagers health).

Rev Chil Pediatr 2010; 81 (6): 515-522

\section{RESUMEN}

Objetivos: Analizar las conductas de autocuidado asociadas a la alimentación y el estado nutricional de niños/ as procedentes de dos colegios de zonas urbanas de la Región Metropolitana, Chile. Su estudio puede cons-

Trabajo recibido el 04 de mayo de 2010, devuelto para corregir el 25 de agosto de 2010, segunda versión el 23 de septiembre de 2010, aceptado para publicación el 27 de septiembre de 2010.

Apoyo económico: Dirección de Investigación, Pontificia Universidad Católica de Chile (DIEE). Escuela de Enfermería, Universidad de George Mason, Fairfax Virginia.

Correspondencia a:

Sonia Jaimovich P.

E-mail: sjaimovi@uc.cl 
tituir un aporte a la prevención de la malnutrición por exceso. Población y Método: Análisis secundario de datos obtenidos en estudio descriptivo y transversal realizado en 2008 en muestra por conveniencia de 207 escolares de 9 a 15 años, que aceptaron participar, en el que se validó el instrumento Moore Index NutritionChild (MIN-C)C. Resultados: Para las conductas estudiadas, el puntaje promedio obtenido por los niños/as en el cuestionario, corresponde sólo al 63\% del ideal (210 puntos). Respuestas que dan cuenta de conductas más desfavorables, dicen relación con obtención de información sobre alimentación saludable a través de diferentes medios y las favorables se relacionan con higiene de la alimentación. La comparación de conductas según estado nutricional no muestra diferencias; el puntaje promedio obtenido por los niños, para toda la escala, fue de 132,2 \pm DS 22,1 (Anova una vía, para efectos fijos). Al analizar los niños con sobrepeso y obesidad en relación con los de estado nutricional normal, las diferencias no son significativas. Discusión y Conclusión: Para contribuir a disminuir la prevalencia del sobrepeso y obesidad, los resultados sugieren intervenciones que privilegien la adquisición de conocimientos y obtención de información.

(Palabras clave: Conductas alimentarias, estado nutricional, salud escolar).

Rev Chil Pediatr 2010; 81 (6): 515-522

\section{Introducción}

El estudio de las conductas de autocuidado asociadas a la nutrición en escolares puede constituir un aporte a la prevención de la malnutrición por exceso; problema multifactorial que no puede ser tratado con métodos convencionales. La literatura señala que las influencias sociales, genéticas y de ambiente familiar compartido, tienen un impacto relevante sobre el patrón de ingesta, la conducta alimentaria y la obesidad infantil ${ }^{1}$.

En los últimos años se observa un aumento significativo del sobrepeso y de la obesidad infantil; en la actualidad es considerado por la OMS la epidemia del siglo ${ }^{2-4}$. En Chile, según cifras del Ministerio de Salud, 2008, el 7,4\% de los menores de 6 años y el 19\% de los escolares de $1^{\circ}$ básico presentan obesidad. La transición nutricional, constituye un riesgo de obesidad y de enfermedades crónicas, influida fuertemente por los cambios en los hábitos de alimentación de la población, en los que gravitan la producción y comercialización de alimentos ${ }^{5}$.

Si bien en términos generales como lo demuestran repetidas encuestas, la población sabe lo que hay que comer para tener una alimentación saludable y también conoce los beneficios de la actividad física, ésta sigue ali- mentándose mal, consumiendo alimentos con alto contenido de azúcar, grasas y sal y manteniéndose muy sedentaria ${ }^{6}$.

Dada la dificultad para lograr cambios de conducta en esta área, ya que es baja la proporción de tratamientos de obesidad efectivos y la mayoría de ellos no perduran en el tiempo ${ }^{7}$, para abordar este problema, el énfasis debe situarse en la promoción de estilos de vida saludable y la prevención.

Disponer de instrumentos que ayuden a diagnosticar las conductas de autocuidado asociadas a la nutrición adquiere hoy día relevancia; el diseño de intervenciones en función de este diagnóstico de conductas, potencialmente, puede contribuir a detener el aumento de la malnutrición por exceso en la población escolar. En este contexto, el año 2008 se realizó un estudio piloto en dos colegios de las zonas urbanas de la Región Metropolitana de Chile. En él se aplicó y validó la versión en español del Moore Index Nutrition-Child (MIN-CC) ${ }^{8}$. Este instrumento ha sido aplicado previamente por sus autoras en un grupo de adolescentes de una comunidad de población ilegal y pobre en Managua, Nicaragua y en estudiantes de cuarto y quinto grado en Washington D.C. En ambas poblaciones se detectó la importancia en la comprensión del impacto de los cambios de conducta en relación a la dieta y mejoraron los 
conocimientos en nutrición de las adolescentes después de la ejecución de un programa educativo sobre nutrición ${ }^{9,10}$.

En el presente artículo se analizan las conductas asociadas a la nutrición referidas por los 207 niños procedentes de 2 colegios en los que se aplica el instrumento; para algunas conductas seleccionadas, se calcula riesgo relativo para niños y niñas y se analizan las respuestas obtenidas, según estado nutricional. Continúa una línea de investigación en el estudio de las prácticas de autocuidado en escolares utilizando instrumentos desarrollados por Moore, J. ${ }^{11,12}$.

\section{Población y Métodos}

Análisis secundario de datos sobre conductas relacionadas con la alimentación, obtenidos de un estudio descriptivo, transversal, realizado en 2008 para validar el instrumento Moore Index Nutrition-Child (MIN-C(C). En este análisis se revisan las conductas, referidas por niños de dos colegios de zonas urbanas de la Región Metropolitana, uno particular del área norte (colegio 1), representativo del nivel socio económico medio alto y otro de la Corporación Belén Educa del área suroriente (colegio 2) representativo del nivel socio económico medio bajo; para estimar el nivel socio económico, se utiliza la ubicación y el tipo de financiamiento de los establecimientos educacionales. El protocolo del estudio piloto, fue aprobado por el comité de ética de la Escuela de Enfermería de la Pontificia Universidad Católica de Chile y el Human Subjects Internal Review Board de la Universidad de Geroge Mason. Contó con la autorización de las autoridades de los establecimientos educacionales y se aplicó un consentimiento informado a padres y niños. En junio de 2008, los niños y padres que aceptan participar en el estudio, completaron el Cuestionario (MIN-CC) y a los niños se les realizó una valoración antropométrica. Participaron niños de ambos sexos de entre 9 y 15 años.

En el estudio piloto, el instrumento MINCC, ya traducido del inglés al español, fue contra traducido al inglés y luego revisado por su autora. En este proceso se aplicaron las recomendaciones planteadas por Carlson, $2000^{13}$, que permiten asegurar una traducción precisa de instrumentos a otro idioma: traducción cuidadosa por un experto en ambos idiomas; contra traducción; prueba preliminar en un grupo pequeño y prueba piloto. Las investigadoras chilenas realizaron las adecuaciones culturales en los ítems en que fue necesario.

El instrumento MIN-CC es un cuestionario con 50 ítems; los resultados referidos a las conductas se analizaron considerando sólo los primeros 42 ítems del instrumento, que tienen una misma escala de medida. Miden la frecuencia de la conducta, variable dependiente analizada, en un rango que va de 1 (nunca) a 5 (siempre) construidos con escala tipo Likert. El puntaje de la escala así considerada oscila entre 42 y 210 puntos. Los puntajes más altos (4 y 5 por ítem) se consideran conductas favorables a una alimentación saludable y los puntajes 1 y 2 como indicadores de conductas de autocuidado desfavorables. Las conductas de autocuidado, se recogieron como variable ordinal.

La validez de contenido del instrumento fue realizada por 11 expertos en enfermería, nutrición, autocuidado y análisis psicométricos (Moore y cols, datos enviados a publicación). La confiabilidad del instrumento, en el estudio piloto referido, fue de coeficiente alpha 0,894 y en su versión en inglés 0,934 .

El procedimiento de aplicación consistió en enviar al domicilio, a través de los niños, una carta con información del proyecto y el consentimiento informado a la muestra por conveniencia de 155 niños del colegio 1 y 150 del colegio 2, para la firma por parte del niño y apoderado, solicitando su devolución en una fecha determinada. En el colegio 1 la facilitadora del proceso fue la profesora de educación física y en el 2 la enfermera del colegio. Se recibió respuesta de 104 y 103 niños respectivamente, por lo que la muestra estudiada quedó conformada por 207 niños, a quienes se les aplicó el cuestionario MIN-C(C) y se les tomaron las medidas antropométricas. El estado nutricional se analizó según Índice de Masa Corporal (IMC) para cada colegio por sexo de acuerdo a CDC-NCHS, que define sobrepeso entre percentil 85 y 95 y obesidad mayor 
a $95^{14}$. En ambos colegios se utilizó la misma metodología para recolectar la información.

Para el plan de tabulación y análisis, el instrumento fue codificado y procesado mediante el programa SPSS versión 17.0 y para los análisis estadísticos se utilizó un alfa de 0,05.

En los resultados, primero se describen las características demográficas de los niños de ambos colegios. Luego se muestra el puntaje promedio obtenido por los niños/as en el instrumento y se compara entre ambos colegios mediante una prueba de Anova de 2 vías de efecto fijo. Para comparar las conductas según estado nutricional (obesidad, sobrepeso y normal) se realiza prueba de Anova una vía, para efectos fijos. Se describen las conductas que constituyen riesgo o protección de una alimentación saludable, referidas por el total de niños. Finalmente, se analizan para cada sexo y estado nutricional, 4 conductas seleccionadas, frente a las cuales las respuestas de los niños fueron neutras, pero que a juicio de las autoras se consideran comportamientos que en nuestro medio pueden favorecer el sobrepeso y la obesidad, determinando en cada de ellas su significancia estadística mediante pruebas de JI cuadrado en tabla de contingencia de $2 \mathrm{x}$ 2. La distribución del estado nutricional y sexo según colegio se realizó mediante pruebas de JI cuadrado en tablas de contingencia multidimensionales.

\section{Resultados}

La población estudiada corresponde a 207 niños chilenos provenientes de 2 colegios de zonas urbanas de la Región Metropolitana, 104 del colegio 1, particular del área norte y 103 del colegio 2 de la Corporación Belén Educa del área suroriente $(50,2 \%$ y $49,8 \%)$. Representan el 67,9\% de los invitados a participar. Con respecto a la distribución por edad y sexo el $58 \%$ de los niños tiene entre 10 y 12 años $(\mathrm{n}=120), 24,6 \% 9$ años $(\mathrm{n}=51)$ y el $9,7 \%(\mathrm{n}=20) 13$ años. El resto, 7,7\% $(\mathrm{n}=16)$ entre 14 y 15 años. El 57,5\% de los escolares son mujeres.

En relación al instrumento MIN-C(C) utilizado para el estudio de las conductas de autocuidado asociadas a la alimentación, los niños de ambos colegios a partir de los 10 años lo respondieron con facilidad en un tiempo de 20 a 30 minutos, algunos menores de 10 años requirieron de ayuda, pero el tiempo de respuesta fue igual y no se comprobaron diferencias en el puntaje obtenido por ellos. Los niños colaboraron al responder el instrumento y manifestaron agrado de participar e interés por los resultados.

El puntaje promedio obtenido por los niños, para los 42 ítems del instrumento analizados, fue de $132,2 \pm 22,1$, cifras que corresponden sólo al 63\% del puntaje ideal (210 puntos). La comparación de los puntajes según estado nutricional de los niños mediante Anova de una vía para efectos fijos, no mostró diferencias entre los niños obesos, con sobrepeso y normales. La prueba de Anova de 2 vías, para las variables colegio y sexo aunque no muestra diferencias significativas, revela levemente una tendencia de puntajes más alto para las niñas (figura 1).

$\mathrm{Al}$ analizar cada conducta en forma separada, se observa que en 11 de las 42 estudiadas, más de la mitad de los niños obtienen 2 o menos puntos, indicador de conductas desfavorables para una alimentación saludable. Por otra parte, para 16 de ellas la mediana está entre 4 y 5 , lo que indica conductas favorables. Entre las respuestas que dan cuenta de conductas más desfavorables, destacan las que dicen relación con obtención de información sobre alimentación saludable a través de diferentes medios (tabla 1). Algunas respuestas que dan cuenta

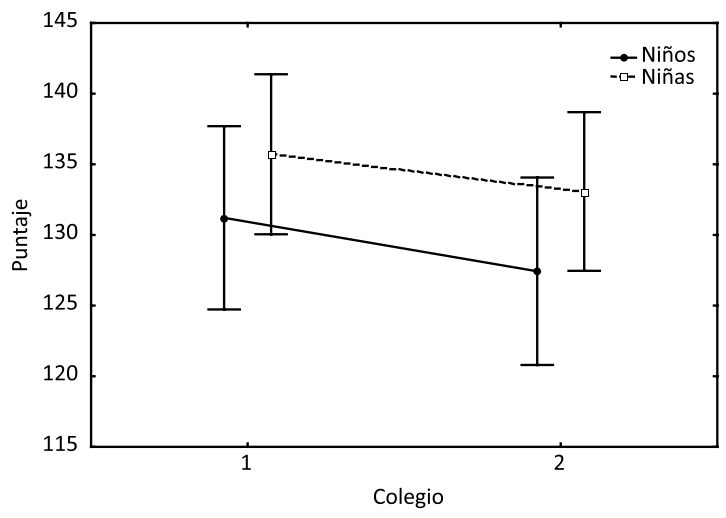

Figura 1. Puntajes promedios (MIN-C C) según sexo y colegio. 
Tabla 1. Conductas identificadas como desfavorables para una alimentación saludable. $\mathbf{n}=207$, junio 2008

\begin{tabular}{|lc|}
\hline Ítems & Mediana \\
\hline 2. Leo sobre nutrición en los libros, revistas u otros medios & 2 \\
\hline 4. Reviso las etiquetas de los alimentos para aprender acerca de los nutrientes en los alimentos & 2 \\
\hline 9. Le pregunto a mi profesor sobre alimentos saludables que debo comer & 1 \\
\hline 13. Le pregunto a mis abuelos sobre alimentación saludable & 2 \\
\hline 19. Estudio nutrición en la escuela & 2 \\
\hline 24. Le hablo a mis amigos acerca de los alimentos saludables que debemos comer & 2 \\
\hline 28. Yo evalúo si mis comidas contienen suficiente proteína & 2 \\
\hline 35. Obtengo información acerca de nutrición en internet & 1 \\
\hline 36. Leo publicidad acerca de alimentos nutritivos & 2 \\
\hline 40. Leo en revistas y periódico sobre los alimentos saludables & 2 \\
42. Pregunto a los otros adultos acerca de los alimentos nutritivos & 2 \\
\hline
\end{tabular}

Tabla 2. Conductas identificadas como favorables para una alimentación saludable. $n=207$, junio 2008

\begin{tabular}{|l|c|}
\hline Ítems & Mediana \\
\hline 8. Pruebo nuevos alimentos & 4 \\
\hline 10. Consumo alimentos que contienen hierro & 4 \\
\hline 11. Elijo comer alimentos que contienen vitaminas & 4 \\
\hline 15. Le pongo mucha sal a los alimentos que consumo & 4 \\
\hline 16. Consumo los mismos alimentos todos los días & 4 \\
\hline 18. Me aseguro de tomar agua limpia & 5 \\
\hline 21. Consumo alimentos que son buena fuente de vitamina C & 4 \\
\hline 22. Lavo las frutas antes de comerlas & 5 \\
\hline 23. Me aseguro que la carne que como está bien cocida & 5 \\
\hline 25. Consumo alimentos que contienen proteínas en cada comida & 4 \\
\hline 26. Trato de comer alimentos y bebidas y que contienen calcio & 4 \\
\hline 27. Consumo alimentos que son buena fuente de Vitamina A & 4 \\
\hline 29. Tomo desayuno todos los días & 5 \\
\hline 31. Prefiero comer dulces en vez de frutas & 4 \\
\hline 33. Tomo café con las comidas & 5 \\
\hline 37. Consumo alimentos variados & 4 \\
\hline
\end{tabular}

de conductas favorables son aquellas que se relacionan con la higiene de la alimentación y las prácticas "tomar desayuno todos los días" y "no consumir café con las comidas" observadas casi en el total de los niños (tabla 2). La prueba Anova de una vía para efectos fijos, no mostró diferencias significativas según estado nutricional de los niños: obesidad, sobre peso y normal $(\mathrm{F}(2,204)=0,859)$; el puntaje promedio obtenido en la escala total tanto por los niños con obesidad y sobrepeso, como por los de estado nutritivo normal, fue 132,2 puntos.

Se estudiaron las diferencias para cada sexo según estado nutricional, en 4 ítems del MINCC que a juicio de las autoras podrían constituir riesgo para sobrepeso y obesidad: 
Tabla 3. Distribución de la población según Estado Nutricional y Colegio. $n=207$, Junio 2008

\begin{tabular}{|lrcrrr|}
\hline IMC & \multicolumn{2}{c}{ Colegio $\mathbf{1}$} & \multicolumn{2}{c|}{ Colegio 2 } & Total \\
& $\mathbf{n}$ & $\mathbf{\%}$ & $\mathbf{n}$ & $\mathbf{\%}$ & \\
\hline Normal & 73 & 70,2 & 62 & 60,2 & 135 \\
\hline Sobrepeso & 26 & 25 & 28 & 27,2 & 54 \\
Obesidad & 5 & 4,8 & 13 & 2,6 & 18 \\
\hline Total & 104 & 100 & 103 & 100 & 207 \\
\hline
\end{tabular}

- Ítem "Elijo tomar bebidas gaseosas en vez de agua" En el análisis separado por sexo, se encontró diferencia significativa JI cuadrado $=10,36 ; \mathrm{gl}=1, \mathrm{p}=0,01)$ en hombres con sobrepeso u obesidad, en comparación con los de estado nutricional normal. En mujeres no se encuentran diferencias significativas.

- Los ítems "Cuando compro una colación elijo una gaseosa en vez de fruta" $y$ "Pienso si lo que como es saludable", no mostraron diferencias significativas en ninguno de los sexos y tampoco constituyeron factor de riesgo en los niños estudiados.

- Ítem "Prefiero comer dulces en vez de frutas" No se encontraron diferencias significativas en hombres ni mujeres con sobrepeso u obesidad, en comparación con los de estado nutricional normal.

Con respecto al estado nutricional, si bien en más del $60 \%$ de los niños es normal, la proporción de malnutrición por exceso es alta. Al analizar con la prueba de JI cuadrado, en tabla de contingencia de $3 \times 2 \times 2$, los niños según colegio, sexo y estado nutricional, las diferencias no son estadísticamente significativas (tablas 3,4 y 5 ).

\section{Discusión y Conclusiones}

En la población de escolares de 9 a 15 años estudiada, se observa que las conductas de autocuidado asociadas a la alimentación no son las ideales para la prevención de la malnutrición por exceso. Esto concuerda con los hallazgos de Vio y Salinas ${ }^{6}$ que comprueban que la población sabe lo que hay que comer
Tabla 4. Prevalencia de obesidad en cifras absolutas: comparación por colegio. Junio 2008, $n=207$

\begin{tabular}{|lcc|}
\hline Estado nutricional IMC & Colegio 1 & Colegio 2 \\
\hline Normal + Sobrepeso & 99 & 90 \\
\hline Obesos* & 5 & 13 \\
\hline
\end{tabular}

${ }^{*} p=0,046 . \quad R R=2,63(I C \quad 0,97-7,10)$

Tabla 5. Prevalencia de sobrepeso y obesidad en cifras absolutas: comparación por colegio. Junio 2008, $n=207$

\begin{tabular}{|lrc|}
\hline Estado nutricional IMC & Colegio 1 & Colegio 2 \\
\hline Normal & 73 & 62 \\
\hline Sobrepeso + Obesos* & 31 & 41 \\
\hline
\end{tabular}

${ }^{*} p=N S 0,13 . R R=1,34($ IC 0,91-1,95)

para tener una alimentación saludable y también conoce los beneficios de la actividad física, pero sigue alimentándose mal, consumiendo alimentos con alto contenido de azúcar, grasas y sal y manteniéndose muy sedentaria. En las conductas estudiadas, se detectan aspectos relacionados principalmente al bajo interés e iniciativa de los niños por buscar información relacionada a una alimentación saludable. Esto podría explicarse, al menos en este grupo de niños por su falta de proactividad que los lleve a desempeñar un papel más protagónico en su cambio de conducta. Viner y Cole ${ }^{15}$ demuestran que los adolescentes activos y cuya alimentación es saludable reducen el riesgo de presentar obesidad en la edad adulta. La literatura señala que las influencias sociales, genéticas y de ambiente familiar compartido, tienen un impacto relevante sobre el patrón de ingesta, la conducta alimentaria y la obesidad infantil $1^{1}$. Otros autores ${ }^{16}$ plantean que la obesidad parece "propagarse a través de las redes sociales", encuentran que el riesgo de convertirse en obesos es un 57\% mayor si la persona tiene un amigo obeso y esta tendencia también se encuentra dentro de las familias. Esto podría interpretarse como un problema en el cual lo conductual tiene mayor peso que lo cognitivo. Los resultados de este análisis no muestran di- 
ferencias en las conductas de los niños según su estado nutricional. A diferencia de lo esperado, los niños con malnutrición por exceso no presentan conductas más desfavorables, lo que podría explicarse porque sus respuestas no reflejan lo que realmente piensan y hacen, o que por su problema nutricional están más preparados para entregar una respuesta socialmente deseable.

Una intervención educativa para la prevención de este problema en la pre-adolescencia y adolescencia, se justifica porque en esta etapa los niños comienzan a decidir sobre los alimentos que consumen y a desarrollar los patrones alimentarios que se traducirán en comportamientos futuros. Larson, NeumarkSztainer, Hannan y Story ${ }^{17}$ encuentran que los adolescentes que comen con sus familias tienden a tener una dieta de mejor calidad y continuar con estos patrones hasta la edad adulta. Crossman, Sullivan y Benin ${ }^{18}$ demuestran que el exceso de peso durante la adolescencia aumenta el riesgo de obesidad durante la edad adulta y que también los hijos de adultos obesos tienen mayor riesgo de presentar este problema de adultos. Estos resultados confirman la utilidad de instrumentos de diagnóstico, como el MIN-C(C), que permitan el diseño de intervenciones para escolares en función de los conocimientos y capacidades detectadas para cada grupo.

Kain, Olivares, Castillo y $\mathrm{Vio}^{19}$ plantean que para lograr los cambios conductuales necesarios para prevenir la obesidad, se requiere en primer lugar adquirir los conocimientos básicos en estos aspectos. Esto es concordante con los resultados de este estudio, ya que las conductas detectadas como desfavorables para una alimentación saludable dicen relación con la adquisición de conocimientos sobre el tema. El interés por buscar información es la etapa inicial para la toma de decisiones y cambio de conducta.

Kain, Uauy, Leyton, Cerda, Olivares y $\mathrm{Vio}^{20}$ demuestran la efectividad de una intervención que incluye educación en alimentación y nutrición y aumento de la actividad física, en la disminución de la obesidad de escolares de la comuna de Casablanca, el cambio observado es mayor en hombres que en mujeres. Si bien los cambios de peso son significativos en los primeros 2 años de seguimiento, la intervención no fue sustentable en el tiempo. Con el fin de lograr la permanencia en el tiempo e influir de forma favorable a la prevención de la obesidad en Chile, los resultados del presente estudio pueden servir de base para el diseño de una intervención que considere la participación de profesores, alumnos y padres en los cambios de conducta necesarios. Entre los objetivos fundamentales propuestos por el Ministerio de Educación de nuestro país para los contenidos de $7^{\circ}$ básico, se encuentra comprender la salud como equilibrio físico, mental y social; y valorar comportamientos relacionados con su preservación ${ }^{21}$. Aunque entre los contenidos para este objetivo no figura explícitamente la alimentación, ésta es parte de los comportamientos que es importante fomentar. En la Unidad 4: Nutrición heterótrofa: procesos de interacción entre sistemas, del mismo documento, aparecen como temas la alimentación en el ser humano, alimentación sana y enfermedades asociadas a la alimentación. El diseño de una intervención educativa basada en las prácticas de autocuidado asociadas a la nutrición de niños permitirá realizar sugerencias concretas que aporten a estos contenidos o a aspectos a desarrollar en cursos previos.

\section{Referencias}

1.- Domínguez-Vásquez P, Olivares S, Santos JL: Influencia familiar sobre la conducta alimentaria y su relación con la obesidad infantil (Eating behavior and childhood obesity: family influences). Arch Latinoam Nutr 2008; 58 (3): 249-55.

2.- Ministerio de Salud: Obesidad en Chile, Un Problema de Salud Pública. En Cumbre de Nutrición y Salud. Chile 2008. Boletín Resultados Cumbre Nutrición y Salud (pdf).

3.- Vio F, Albala C: Epidemiología de la Obesidad en Chile. Rev Chil Nutr 2000; 27: 97-104.

4.- Flegal $K$ : The obesity epidemic in children and adults: current evidence and research ideas. Med Sci Sports Exerc 1999; 31: 509-14.

5.- Vio F, Albala C, Crovetto M: Promoción de Salud en la Transición Epidemiológica de Chile. Rev Chil Nutr 2000; 27: 21-9. 
6.- Vio F, Salinas J: Promoción de Salud y Calidad de Vida en Chile: Una política con nuevos desafíos. Rev Chil Nutr Octubre 2006; Vol. 33, Suplemento $\mathrm{N}^{\circ} 1$.

7.- Moraga F, Rebollo, MJ, Bórquez P, Cáceres J, Castillo $C$ : Tratamiento de la obesidad infantil: Factores pronósticos asociados a una respuesta favorable. Rev Chil Pediatr 2003; 74 (4): 374-80.

8.- Moore JB, Pawloski L, Baghi H, et al: Development and examination of psychometric properties of selfcare instruments to measure nutrition practices for English and Spanish-speaking adolescents. Self-Care, Dependent-Care, and Nursing: The Official Journal of the International Orem Society 2005; 13 (1): 9-16.

9.- Moore JB, Pawloski LR, Rodriguez C, Baghi H, Lumbi $L$, Zamora $L$ : The effects of a nutrition education program on the nutrition self-care practices of Nicaraguan adolescent girls and the nutrition dependent-care practices of their mothers. Self Care Dependent Care Nurs 2007; 15: 6-11.

10.- Moore JB, Pawloski, LR, Goldberg P, Oh KY, Stoehr A, Baghi H: Childhood Obesity Study: A Pilot Study of the Effect of the Nutrition Education Program Color My Pyramid on Nutrition Knowledge, Self-Care Practices, Physical Activity, and Status. J School Nurs 2009; 25: 230-9.

11.- Campos MS, Campos C, Jaimovich S: Prácticas de Autocuidado de escolares. Revista EPAS 1999, Número especial, 77-81.

12.- Jaimovich S, Campos C, Campos MS, Moore J: Spanish Version of the Child and Adolescent Self-Care Performance Questionnaire: Psychometric Testing. Pediatr Nurs 2009; 35 (2): 109-14.

13.- Carlson ED: A case study in translation methodology using the Health-Promotion Lifestyle Profile II. 2000, vol. 17, no1, pp. 61-70 Public Health; United States.

14.- Kuczmarski RJ, Ogden CL, Grummer-Strawn LM, et al: CDC growth charts: United States. Advance data from vital and health statistics. Hyattsville, MD: National Center for Health Statistics; 2000; no. 314.

15.- Viner RM, Cole TJ: Who changes body mass between adolescence and adulthood? Factors predicting change in BMI between 16 years and 30 tears in 1970 British Birth Cohort. Int J OBES 2006; 30: 1368-74.

16.- Christakis N, Fowler J: The Spread of Obesity in a Large Social Network over 32 Years. N Engl J Med 2007; (4) $357: 370-9$.

17.- Larson NI, Neumark-Sztainer D, Hannan PJ, Story M: Family meals during adolescence are associated with higher diet quality and healthful meal patterns during young adulthood. J Am Diet Assoc 2007; 107: 1502-10.

18.- Crossman A, Sullivan AD, Benin M: The family environment and American adolescents' risk of obesity as young adults. Soc Sci Med Volume 63, Issue 9, November 2006, p 2255-67.

19.- Kain J, Olivares S, Castillo M, Vio F: Validación y aplicación de instrumentos para evaluar intervenciones educativas en obesidad en escolares. Rev Chil Pediatr 2001; 72 (4); 308-18.

20.- Kain J, Uauy R, Leyton D, Cerda R, Olivares S, Vio F: Efectividad de una intervención en educación alimentaria y actividad física para prevenir obesidad en escolares de la Ciudad de Casablanca, Chile (2003-2004). Rev Med Chile 2008; 136: 22-30.

21.- Gobierno de Chile, Ministerio de Educación: Estudio y Comprensión de la Naturaleza. Programa de Estudio Séptimo Año Básico / Nivel Básico 5. Educación Básica, Unidad de Curriculum y Evaluación, 2004. ISBN 956-7933-50-2. 